\section{P3-S1.26 NOVEL CHLAMYDIA TRACHOMATIS POINT OF CARE RAPID TEST SHOWS DISAPPOINTINGLY LOW CLINICAL SENSITIVITY IN UROGENITAL CHLAMYDIA INFECTIONS IN PARAMARIBO, SURINAME}

doi:10.1136/sextrans-2011-050108.426

\begin{abstract}
${ }^{1} \mathrm{~J}$ van der Helm, ${ }^{2} \mathrm{~L}$ Sabajo, ${ }^{3} \mathrm{~S}$ Morré, ${ }^{4} \mathrm{~A}$ Grunberg, ${ }^{1} \mathrm{~A}$ Speksnijder, ${ }^{5} \mathrm{H}$ de Vries. ${ }^{1}$ Health Service Amsterdam, Amsterdam, Netherlands; ${ }^{2}$ Dermatology Service, Ministry of Health, Suriname; ${ }^{3} \mathrm{VU}$ University Medical Center, Netherlands; ${ }^{4}$ Lobi Foundation, Suriname; ${ }^{5}$ Health Service Amsterdam, Academic Medical Center, University of Amsterdam, Netherlands
\end{abstract}

Background Affordable and reliable point of care (POC) tests to diagnose urogenital chlamydia infections (POC-Ct) are needed, especially in resource limited settings. WHO has formulated standards that POC tests have to meet. One of those is that the test should be sensitive. Three POC-Ct tests currently on the market all showed poor sensitivity between $12 \%$ and $17 \%$ in a nonmanufacturer sponsored clinical study (van Dommelen 2010). One POC-Ct test evaluated in a manufacturer-sponsored study claims over $80 \%$ sensitivity (Mahilum-Tapay 2007). We evaluated the performance of this POC-Ct in two outpatient clinics in Suriname, S.A.

Methods Between July 2009 and February 2010963 women were included in a high risk STI clinic $(\mathrm{n}=181)$ and a low risk birth control clinic $(n=782)$ in Paramaribo, Suriname. Nurse collected vaginal swabs were obtained for the POC-Ct (Diagnostics for the Real World, LTD, Cambridge, UK) and control NAAT (APTIMA Combo 2, Gen-Probe, San Diego, USA) in a cross-over model. Swabs were processed according to the manufacturers instructions. POCCt was compared to NAAT and sensitivity, specificity, positive- and negative predictive value (PPV, NPV) were calculated. Quantitative Ct load was determined with a real-time PCR targeting the cryptic plasmid. Ct load was expressed as inclusion forming units (IFU) based on defined serial dilutions. An independent t-test was used to compare log-transformed $\mathrm{Ct}$ loads between true positive and false negative POC-Ct results.

Results Ct prevalence, determined by NAAT, was 23\% at the high risk STI clinic and 9\% at the low risk birth control clinic. Four samples were excluded due to discrepancy in POC-Ct result between two lab technicians $(n=3)$ and failure of POC-Ct $(n=1)$. Performance results of POC-Ct compared to NAAT are shown in Abstract P3-S1.26 table 1. Quantitative Ct bacterial load was 65 times higher when POC-Ct detected Ct infection (geometric mean 115 IFU) compared to loads that POC-Ct did not detect (geometric mean 1.8 IFU, $p<0.001$ ). Human DNA concentration did not differ between the true positive and false negative POC-Ct results $(p=0.904)$. Sensitivity of POC-Ct in samples with low Ct load was $16 \%$.

Abstract P3-S1.26 Table 1 Performance results of POC-Ct compared to NAAT

\begin{tabular}{llcllll}
\hline & NAAT + & NAAT - & Sensitivity & Specificity & PPV & NPV \\
\hline POC-Ct + & 48 & 30 & $41.7 \%$ & $97.6 \%$ & $61.5 \%$ & $92.4 \%$ \\
POC-Ct - & 67 & 814 & & & &
\end{tabular}

Conclusion The sensitivity and to a lesser extend the PPV of the POC-Ct did not meet the expectations as described previously (83.5\%). The POC-Ct missed samples with a low Ct load. With a sensitivity of $41.7 \%$ the Diagnostics of the Real World POC-Ct test does not meet the ASSURED criteria of a sensitive test formulated by the WHO.

\section{P3-S1.27 IS ANTIMICROBIAL RESISTANCE IN CHLAMYDIA TRACHOMATIS A REALITY?}

doi:10.1136/sextrans-2011-050108.427

${ }^{1} \mathrm{R}$ Pitt, ${ }^{1} \mathrm{~S}$ Alexander, ${ }^{2} \mathrm{P}$ Horner, ${ }^{1} \mathrm{C}$ Ison. ${ }^{1}$ Health Protection Agency, CfI, London, UK; ${ }^{2}$ University of Bristol, UK

Introduction The use of single dose therapy and the emergence of a stable tetracycline resistant strain of Chlamydia suis has raised concerns regarding potential development of antimicrobial resistance (AMR) in C trachomatis (CT). Specimens sourced from patients with persistently confirmed CT infections after treatment with first line recommended therapies were examined for the presence of AMR determinants identified in other organisms.

Methods CT DNA positive specimens were examined for viable bacteria by tissue culture. Sequencing for mutations in three genes known to be involved in azithromycin (AZM) AMR; rplV, rplD and 23S rRNA (two alleles) and for the presence of six tetracycline AMR determinants; tet $(A)$, tet $(B)$, tet $(C)$, tet $(D)$, tet $(\mathrm{E})$ and tet $(M)$ was done. Susceptibility testing was performed using isolates grown in duplicate serial dilutions of AZM followed by quantitative RT-PCR. Isolates with a log or more increase in plasmid copy number were deemed resistant and those with a static or decreased copy number were deemed sensitive. Isolates were also screened for contaminating bacteria.

Results Nine isolates were retrieved from twenty CT NAAT positive patients. When examined by AZM MIC assays, all isolates were sensitive. An identical non-silent single nucleotide polymorphism (SNP) was identified in three clinical specimens and one isolate from three patients in the rplD gene. A non-silent SNP was also identified in the rplV gene in one of these patients. The pre and post treatment specimen from a further patient showed a non-silent SNP in the rplD gene and identical point mutations in both alleles of the 23S rRNA gene. The tet $(M)$ resistance determinant was identified in ten specimens and five isolates from eleven different patients. All screens for contaminating bacteria were negative.

Conclusions Previously unreported mutations in genes responsible for AZM AMR in other organisms have been identified however all isolates were found to be AZM sensitive. Whilst the SNPs identified may not be significant, detection of them demonstrates that CT has undergone mutation in these key genes. A fragment of the tetracycline AMR determinant, tet $(M)$ (responsible for high level tetracycline AMR in Neisseria gonorrhoeae), was identified in several clinical specimens and in five isolates. The function of the tet $(M)$ fragment in CT is currently unknown and the establishment of tetracycline MIC assays are a priority.

\section{P3-S1.28 IS URETHRITIS OF UNKNOWN AETIOLOGY CAUSED BY BACTERIA ASSOCIATED WITH BACTERIAL VAGINOSIS?}

doi:10.1136/sextrans-2011-050108.428

${ }^{1} \mathrm{M}$ Frølund, ${ }^{1} \mathrm{R}$ Datcu, ${ }^{1} \mathrm{P}$ Ahrens, ${ }^{2} \mathrm{P}$ Lidbrink, ${ }^{2} \mathrm{E}$ Björnelius, ${ }^{1} \mathrm{~J} \mathrm{~S}$ Jensen. ${ }^{1}$ Statens Serum Institute, Copenhagen S, Denmark; ${ }^{2}$ Karolinska University Hospital, Sweden

Background The aetiology of non-gonococcal urethritis (NGU) in men is unknown in $30 \%-50 \%$ of cases. Little is known about the relation of bacterial vaginosis (BV) associated bacteria in men with urethritis of unknown aetiology (UUE). Urethral swabs from men with and without NGU were analysed with PCR for BV associated bacteria to show a possible association with UUE.

Methods Urethral swabs from 9 and 19 men with symptomatic and asymptomatic NGU ( $>5 \mathrm{PMNL} / \mathrm{hpf}$ ), respectively, and 30 asymptomatic men without NGU were collected. All samples were negative for Neisseria gonorrhoeae, Chlamydia trachomatis, Mycoplasma genitalium, Ureaplasma urealyticum and U parvum with specific PCR assays. Quantitative real-time PCR with TaqMan based assays were 